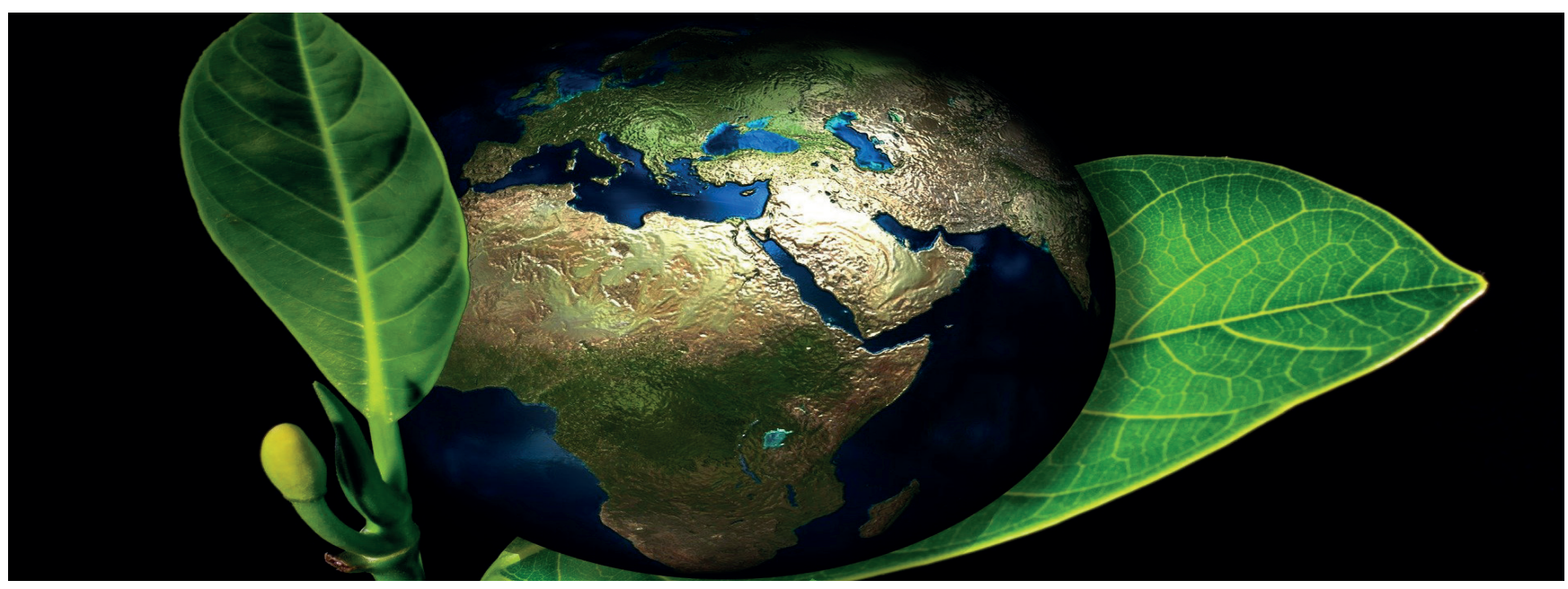

Tomado de: https://pixabay.com/es/illustrations/tierra-globo-nacimiento-nuevo-405096/

\title{
La criminología verde como elemento fundamental en la formación profesional de criminólogos en México ${ }^{1}$
}

\section{Green criminology as a fundamental element in professional training of criminologists in Mexico}

José Luis Carpio Domínguez*, Inés Arroyo Quiroz, Cynthia Marisol Vargas Orozco

\section{RESUMEN}

El presente estudio tiene como objetivo reflexionar sobre la contribución de los estudios de la educación en Criminología en México para abordar crímenes y daños socio ambientales actuales. Para lograr dicho objetivo, primero se llevó a cabo una revisión documental de los aportes educativos, teóricos y metodológicos que ha hecho la criminología en el estudio de los crímenes ambientales, además se revisaron los planes de estudio de las universidades que imparten la licenciatura en Criminología, tomando en consideración los siguientes conceptos clave: naturaleza y medio ambiente, ecología, desarrollo sustentable/sostenible, bioética y ciencias ambientales. En la revisión se encontraron dos resultados principales: únicamente el $8.3 \%$ de las universidades que imparten la licenciatura en Criminología incluyen en sus planes de estudio algún tema sobre problemáticas socioambientales y no existe ninguna materia en los planes de estudios que contemple los crímenes ambientales como unidad de estudio. Se concluye que el estudio y análisis de los crímenes ambientales es aún una tarea pendiente en la formación profesional de criminólogos en México, por lo que se propone el contenido temático para una materia sobre Criminología Verde en la formación profesional de criminólogos en el país.

Palabras clave: criminología verde, crímenes ambientales, educación ambienta.

\section{ABSTRACT}

The purpose of this study is to reflect on the contribution of criminology education studies in Mexico to address current socio-environmental crimes and damages. To achieve this objective, first a documentary review of educative, theoretical and methodological contributions that criminology has made in the study of environmental crimes will be carried out, in addition the programs of the universities that teach the degree in Criminology were reviewed considering the following key concepts: nature and environment, ecology, sustainable development, bioethics and environmental sciences. In the review, two main results were found: $8.3 \%$ of the universities that offer a degree in Criminology include in their curriculum some topic on socio-environmental problems but there was no subject on environmental crimes as a unit of study. It is concluded that the study and analysis of environmental crimes is still a pending task in the professional training of criminologists in Mexico, so we propose a thematic content for a subject matter on Green Criminology for the training of these professionals.

Keywords: green criminology, environmental crimes, environmental education.

${ }^{1}$ Este documento es resultado de una parte de la exploración de literatura del proyecto de tesis doctoral en CienciasSociales con Orientación en Desarrollo Sustentable del Instituto de Investigaciones Sociales de la Universidad Autónoma de Nuevo León y en colaboración con miembros del Cuerpo Académico en Criminología de la Unidad Académica Multidisciplinaria Reynosa Aztlán de la Universidad Autónoma de Tamaulipas y el Centro Regional de Investigaciones Multidisciplinarias de la UNAM. Fecha de recepción: 30.05.2019 Fecha de aceptación: 20.06.2019

*Autor para correspondencia: jcarpio@docentes.uat.edu.mx Dirección: Unidad Académica Multidisciplinaria Reynosa Aztlán de la Universidad Autónoma de Tamaulipas 


\section{INTRODUCCIÓN}

El estudio de las problemáticas ambientales es de carácter transversal, la heterogeneidad de disciplinas que las abordan ha permitido la comprensión, origen y atención de los desafíos de la protección del medio ambiente a escala global y la criminología no ha sido la excepción, las primeras aportaciones de la disciplina a nivel internacional han marcado una pauta de actuación científica, educativa y pragmática con relación a los desafíos actuales, incluyendo aquellos que se relacionan con el medio ambiente.

Un aspecto importante es que en México los esfuerzos en el tema ambiental desde la criminología se han originado dentro del contexto de investigación científica, quedando pendiente la educación de estos profesionistas como un recurso de capital social. Los esfuerzos, aunque más o menos direccionados, necesitan un eje articulador entre dos temas que parecen a primera vista muy distantes uno de otro: la criminología y el medio ambiente.

La educación ambiental, como un recurso social, es un medio para lograr el desarrollo sustentable (Fernández y Gutiérrez, 2013) y la universidad es la encargada de formar perfiles profesionales adecuados a las necesidades sociales y adecuados a la realidad social e histórica, es en estas instituciones donde los estudiantes perfilan una postura profesional ante la realidad fuera del aula, determinando en gran medida las percepciones que éstos tengan del ambiente que los rodea (social o natural) y en el que desarrollan su vida personal y profesional; por lo que las universidades deben asumir un rol proactivo complementando las acciones de otras instituciones del gobierno, las organizaciones no gubernamentales y las empresas, para liderar programas de formación, difusión y extensión del conocimiento (Perdomo y Escobar, 2011). La problemática planteada propone una revisión de la importancia de las problemáticas ambientales en la enseñanza criminológica en las universidades a través de los métodos científicos y prácticos de esta disciplina, sabiendo que el análisis de los crímenes ambientales (desde la visión académica o laboral) como parte del objeto de estudio de la criminología representan un tema de estudio pertinente y actual adecuado a la realidad socio-histórica global, regional y nacional; ante esto surge la pregunta ¿La formación académica en criminología en México comprende también el estudio de las principales problemáticas ambientales como parte del perfil profesional? Para responder esta cuestión, el presente ensayo plantea los siguientes objetivos:

1. Llevar a cabo una revisión diacrónica documental de los aportes y la evolución de la criminología verde en México.

2. Identificar el contenido de materias relativas a crímenes ambientales en la enseñanza de la Criminología en el país a nivel educativo superior.

3. Proponer una materia, de nivel universitario, que aborde la problemática de crímenes ambientales tomando como marco de referencia la Criminología Verde. Cada uno de estos objetivos da lugar a respectivas secciones de este trabajo:

\section{Etapa 1: Aportes teóricos y metodológicos que} ha hecho la criminología al estudio de los crímenes ambientales en México.

En esta etapa se realizó una sístesis del origen de la educación ambiental y a través de una revisión diacrónica del surgimiento y evolución de la criminología verde en México, basada en consultas de bibliografía especializada en bases de datos de revistas científicas y registros hemerográficos se llevó a cabo un análisis documental que permitió vincular la perspectiva verde en la criminología con la educación ambiental con el fin de contextualizar a esta disciplina en los estudios ambientales.

Etapa 2: Contenido de materias educativas relativas a crímenes ambientales en la enseñanza de la Criminología en el nivel educativo superior del país

Se consultó la base de datos de la Asociación Nacional de Universidades e Instituciones de Educación Superior (ANUIES, 2019) en donde se identificaron 96 universidades que im- 
parten Criminología: 8 del sector público y 88 del sector privado, incluyendo aquellos pregrados multidisciplinarios como Criminalística, Ciencias Forenses, Seguridad Pública, ya que en algunas universidades suelen aparecer dos disciplinas en una misma licenciatura (p.ej. Licenciado en Criminología y Criminalística). Se tomó como muestra de análisis un plan de estudios por universidad para evitar réplicas de información, ya que cada universidad cuenta con planes de estudio homologados para varias sedes en diferentes ciudades de la república.

Una vez identificados los programas educativos, se llevó a cabo una consulta en los planes de estudio acerca de materias relativas a las siguientes temáticas: Naturaleza, Medio Ambiente, Bioética, Desarrollo Sustentable/Sostenible y Ciencias Ambientales que permitan poner en perspectiva la educación ambiental dentro de la formación profesional en Criminología. Se llevó a cabo la consulta de las variables directamente en los portales digitales de las universidades y por vía telefónica, esta información se colectó y procesó, tomando en cuenta las variables mencionadas anteriormente.

Etapa 3: Propuesta de una materia a nivel universitario que aborde la problemática de crímenes ambientales tomando como marco de referencia la Criminología Verde.

En la tercera y última etapa se realizó una triangulación de la información de la consulta de los planes de estudio y los aportes teóricos y metodológicos de la criminología verde en el país, derivando en una propuesta de materia para los planes de estudio en criminología, que sirva de apoyo tanto para las futuras reformas como para unidades de aprendizaje en la enseñanza criminológica, que contemple los principales focos de atención de la realidad ambiental y fomente la discusión teórica y el alcance de las nuevas perspectivas de la criminología.

\section{DESARROLLO}

Marco de Referencia: el origen de la educación ambiental y la criminología verde
El medio ambiente ha sido siempre esencial para la vida, en tanto que el desarrollo biológico y evolutivo de las especies animales y vegetales y demás organismos que lo componen dependen de su calidad, integridad y buen manejo. En la carrera evolutiva los humanos han destacado sobre otras especies, desarrollando una inteligencia (evolución cultural) que les permitió adaptarse o modificar su entorno para sobrevivir (Márquez, 2002).

Fue el crecimiento de la población humana y su adaptabilidad a diferentes ambientes y climas, lo que los colocó en una situación de ventaja frente a otras especies y en el transcurrir de los años se convirtió en una especie dominante a nivel global. Sin embargo, fue hasta la década de los cincuenta cuando el hombre comienza a crear conciencia sobre el mundo en el que vive y el impacto que ha provocado su desarrollo como especie en el planeta. Los trabajos de Carson (1962) y Hardin (1968) comenzaron a romper algunos paradigmas relacionados al uso desmedido de la naturaleza para el beneficio humano y a analizar cómo las actividades humanas dañaban el medio ambiente, sin embargo, fue hasta 1972, durante la Conferencia de las Naciones Unidas sobre el Medio Humano (Estocolmo, Suecia) que se analizaron con mayor precisión los efectos de las actividades humanas sobre ambientes biofísicos, flora y fauna silvestre, suelo y agua; efectos de escala global que sin duda debían ser atendidos.

De acuerdo con el Programa de las Naciones Unidas para el Ambiente (PNUMA, 2002) la Conferencia de Estocolmo articuló el derecho de las personas a vivir en un medio ambiente de calidad que les permita llevar una vida digna y gozar de bienestar y una vez que se estableció este derecho, alrededor de 50 gobiernos de todo el mundo adoptaron instrumentos y constituciones que reconocían al medio ambiente como un derecho humano fundamental. A partir de dicha conferencia, se desarrollaron gran parte de las legislaciones nacionales relativas al medio ambiente y los temas ambientales se posicionaron en un gran número de agendas nacio- 
nales y regionales (Clarke y Timberlake, 1982).

Durante la década de los ochenta, el Informe Bruntland (1987) reconoció el deterioro ambiental a escala global, manifestado a través de pérdida de biodiversidad y ecosistemas, aumento en la extinción de especies y contaminación de suelo y agua, entre otros indicadores ambientales, y dejó claro que las actividades humanas rebasan la capacidad del planeta para regenerar los recursos que se utilizan y para absorber lo que se desecha.

A partir de entonces, se han ido desarrollando a nivel global diferentes convenciones, movimientos y legislaciones encaminados a defender el medio ambiente y la calidad de vida de los humanos, considerando aspectos como el cambio climático global, la protección y conservación de flora y fauna silvestres, conservación y uso razonable de los recursos ambientales en beneficio humano, entre otros aspectos (Global Footprint Network, 2013).

La postura sobre el cuidado al medio ambiente está relacionada con la manera en que se entiende al ser humano en relación con la naturaleza (PNUMA, 2002) y generalmente se parte de dos perspectivas: 1. antropocéntrica, que contempla al hombre como el centro del discurso medioambiental y 2. ecocéntrica, en la que el ser humano representa un miembro más del nicho ecológico (biósfera) en donde desenvuelve su vida y sus actividades (Thompson y Barton, 1994). En este sentido, han sido diversos los aportes que se han hecho para regular el impacto de las actividades humanas en busca del desarrollo del medio ambiente. Así, desde el siglo pasado, se planteó la necesidad de recurrir a la educación ambiental como una estrategia para incidir en las decisiones institucionales, económicas, políticas, ecológicas y sociales para la protección y uso responsable del medio ambiente (Giordan y Souchon, 1995; Montemayor, 2003). Entre los grandes hitos de la educación ambiental se pueden mencionar, entre otros: 1) la Declaración de Estocolmo (1972) en la que se establece en el Principio 19: Es indispensa- ble una educación en labores ambientales dirigida tanto a las generaciones jóvenes como los adultos y que se preste la debida atención al sector de la población menos privilegiada para ensanchar las bases de la opinión pública bien informada y de una conducta de los individuos, de las empresas y de las colectividades; inspirada en el sentido de su responsabilidad en cuanto a la protección y mejoramiento del medio en toda su dimensión humana (ONU, 1972); 2) la "Carta de Belgrado" (1975), en la que se concibe a la educación ambiental como una herramienta que contribuye a la formación de una nueva ética del planeta, reconoce las interrelaciones del hombre-sociedad-naturaleza y la necesidad de realizar transformaciones en las políticas nacionales hacia una distribución equitativa de las reservas mundiales, de recursos naturales y la satisfacción de las necesidades de todos los países (PNUMA, 1975); y, 3) la Agenda 21 y el Foro Global Ciudadano (Cumbre de Río, 1992), los cuales reorientan la educación hacia el desarrollo sostenible y establecen a la educación ambiental como un acto para la transformación social y un proceso de aprendizaje permanente basado en el respeto a todas las formas de vida (ONU, 2000). Desde de la corriente humanista (Sauvé, 2004), la educación ambiental realiza un análisis y una crítica a las dinámicas sociales que influyen en las realidades y problemáticas ambientales como mecanismo de evaluación, concientización y acción que busca a través de la educación generar responsabilidad social e institucional ante el desafío del uso racional del medio ambiente.

En la actualidad, la educación ambiental constituye un nuevo enfoque pedagógico que se define como un proceso que forma al individuo para desempeñar un papel crítico en la sociedad, con objeto de establecer una relación armónica con la naturaleza, brindándole elementos que le permitan analizar la problemática ambiental actual y conocer el papel que juega en la transformación de la sociedad, a fin de alcanzar mejores condiciones de vida. Asimismo, es un proceso de formación de actitudes y valores para el compromiso social (Secretaría de Me- 
dio Ambiente y Recursos Naturales (Otero, 2000; Montemayor, 2003; SEMARNAT, 2018). Sin duda, la educación es un factor potencial de transformación social que permite la multidireccionalidad de los diferentes grupos sociales que ha ido adaptándose a la realidad sociohistórica, y partiendo de su base científica, rompe y construye paradigmas conforme adquiere y genera nuevos conocimientos sobre la realidad y a su vez permite la dispersión del conocimiento.

\section{Criminología Verde}

La criminología desde la década de 1990 tuvo aportes significativos a las problemáticas ambientales, tomando dichos problemas como consecuencia de actividades humanas que se configuraban como "crímenes" contra el medio ambiente, fue entonces que Lynch (1990) introduce el término "Green Criminology" y a pesar de varios debates sobre la construcción terminológica de esta nueva perspectiva de la criminología fueron los criminólogos británicos Clifford y Edwards (1998), Groombridge (1998), Lane (1998) y Sur (1998) quienes sugirieron la formación del término "criminología verde".

Esta perspectiva se formó a partir de las discusiones sobre los estudios criminológicos de criminalidad ecológica por miembros de la élite social (Eman, Mesko y Fields, 2009), por lo que investigadores como Carrabine, Iganski, Lee, Plummer y South (2004) establecieron que existen cuatro líneas principales que debe atender la "Green Criminology": primero investigar la existencia de crímenes "verdes" o contra el medio ambiente en todas sus formas y el desarrollo de tipologías y diferencias básicas, identificar las diferentes direcciones en este campo y evaluar las complicaciones e influencias políticas, conectar los delitos verdes con las desigualdades sociales, y por último evaluar esta nueva perspectiva en los cambios sociales que vayan sucediendo.

En este sentido, Eman, Mesko y Fields (2009) establecen que "atender los crímenes ambientales en la actualidad representa uno de los mayores desafíos para la teoría y práctica ju- rídica, legislación penal, criminología, sociología, ecología, criminalística, victimología y prevención del delito y a su vez en la construcción terminológica de los crímenes ambientales que han sido definidos como: criminalidad del medio ambiente, criminalidad ambiental, ecocidio, delitos verdes, etc.” (p. 578).

Para Pecar (1981) un crimen ambiental es todo acto o proceso permanente o temporal que tiene una influencia negativa sobre el medio ambiente, la salud de las personas o los recursos naturales, incluso la construcción, cambio, abandono y destrucción de edificios; los residuos su procesamiento y eliminación; transporte y manejo de sustancias peligrosas; dañar o destruir los recursos naturales; la reducción de la diversidad biológica o reducción de los recursos genéticos naturales; y otras actividades o intervenciones que ponen en riesgo el medio ambiente (p. 40). Además, caracterizó la contaminación de la naturaleza y el medio ambiente como una devaluación del medio ambiente, lo que también denominó "ecocidio", término para definir la destrucción intencional del entorno vital y que en México es definido como:

Conducta dolosa determinada por las normas penales, consistente en causar un daño grave al ambiente por la emisión de contaminantes, la realización de actividades riesgosas o la afectación de recursos naturales, en contravención a lo dispuesto en la presente ley o en las normas oficiales ambientales mexicanas (Ley Ambiental de Protección a la Tierra en el Distrito Federal, 2017; Artículo 5, p. 9)

Además, Pecar (1988) definió que el crimen ambiental es el resultado del egoísmo, que está determinado por la necesidad, con fines de lucro asociados al control de la naturaleza (citado por Eman, Mesko y Fields, 2009) y para Clifford (1998) el delito ambiental es un acto que se comete con la intención de dañar el medio ambiente y su sistema ecológico y biológico para asegurar el beneficio empresarial o personal. Por lo que la construcción conceptual, teórica y 
práctica se prolonga hasta la actualidad, en los últimos veinte años varios investigadores han aportado y generado conocimientos importantes relativos al tema, entre los que se encuentran Groombridge (1998), Beirne (1999), Lynch y Stretesky (2003), Walters (2006), Beirne y South (2007), Benton (2007), Hauck (2008), Sollund (2008), White (2008), Croall (2009), Eman, Meskoy Fields (2009), Gibbs etal. (2010), Katz (2010), Van Solinge (2010), Ruggiero y South (2010), Bisschop (2012), Agnew (2012), Jarrell y Ozymy (2012), Wyatt (2012), Nurse (2013), Hall (2013), South y Brisman (2013) y Takemura (2015), entre otros que han hecho considerables aportes a la criminología desde la perspectiva ambiental. Aportes educativos, teóricos y metodológicos que ha hecho la criminología en el estudio de los crímenes ambientales en México.

En México, la participación de la criminología ha procurado atender problemáticas de diversa índole, sobre todo aquellas que amenazan la seguridad nacional como los distintos tipos de tráfico (narcóticos, personas y armas), delincuencia organizada, narcoterrorismo, homicidios, secuestro, entre otros, por otra parte, los aportes de la criminología en los temas ambientales han ocupado un lugar periférico dentro de las estrategias de actuación y generalmente se ha abordado el tema desde las disciplinas de las ciencias naturales, pero siempre presentes en las agendas gubernamentales del país.

La criminología en México es definida como "una ciencia sintética, causal-explicativa, natural y cultural de las conductas antisociales" (Rodríguez, 2015, p.5) por lo que su objeto de estudio, las conductas antisociales, son entendidas como aquellas conductas humanas que van contra el bien común, atentan contra la estructura básica de la sociedad, destruye sus valores fundamentales y lesiona las normas de convivencia (p. 25).

A su vez, para la criminología el fenómeno antisocial puede analizarse en diferentes niveles y cada uno con sus marcos teóricos y metodológicos particulares, estos niveles son llamados "niveles de interpretación criminológica": crimen, criminal y criminalidad (Pinatel, 1960, citado en Rodríguez, 2015, p.29). A). Crimen es la conducta antisocial, es un episodio que tiene una temporalidad (principio, desarrollo y fin) en este nivel se analizan todos los factores y causas que apoyaron la manifestación de la conducta. B). Criminal es el autor del crimen y no se limita a la percepción legal (delincuente) del individuo antisocial, es decir, no todos los criminales son delincuentes y viceversa, por último, C). Criminalidad es el conjunto de conductas antisociales que se producen en un tiempo y lugar determinado, su estudio consiste en un análisis global del fenómeno, pero generalmente va acompañada de un reduccionismo de información ya que generalmente se fundamenta en datos $y$ cifras oficiales de las instituciones, las cuales permiten un análisis muy parcializado de los fenómenos antisociales.

En este sentido, la perspectiva verde de la criminología en México tiene un campo de acción aún en exploración, pero parte de la búsqueda de un concepto unificado que permita definir los crímenes ambientales desde la postura social, ambiental y económica como elementos intrínsecos de convergencia del fenómeno antisocial en el medio ambiente para lograr su prevención, de manera previa, Carpio et al. (2018) establecen que "un crimen ambiental es toda conducta antisocial que menoscaba la integridad ecológica y el patrimonio colectivo, de manera no controlada, y en consecuencia fuera de la ley, limitando las capacidades de productividad de las comunidades y las regiones" (p. 32) término enfocado a la sustracción y destrucción de flora, fauna y ecosistemas.

En México, los trabajos de Sosa (2011) en el análisis de la aplicación de la ley a casos de tráfico de especies, Alvarado (2012) desde la generación de conocimiento sobre la participación de la delincuencia organizada en los delitos ambientales, los aportes de Toledo (2015) sobre el ecocidio en México, Carpio et al. (2018) en el estudio de las nuevas redes de tráfico de es- 
pecies, Arroyo y Wyatt (2018) en el análisis de casos de crímenes ambientales en el país y los trabajos de Peralta (2018) sobre los tribunales verdes, y que desde disciplinas como el derecho, economía, ecología, biología, etc., han aclarado el camino de la criminología y las ciencias afines en el abordaje de los problemas ambientales desde la perspectiva social, económica y legal.

En cuanto a los aportes educativos en el tema, en el año 2016, se organizó el curso "Green Criminology: An Introduction”, organizado de manera conjunta entre el Centro Regional de Investigaciones Multidisciplinarias de la Universidad Nacional Autónoma de México y la Northumbria University de Inglaterra en la ciudad de Cuernavaca, Morelos; del cual se derivó como producto de docencia el libro "Criminología Verde en México”, publicado en ambos países, estableciendo el primer vínculo entre la criminología y la problemática ambiental en el país.

En febrero de 2019 se dio inicio al diplomado a distancia "Criminología y Victimología" organizado por el Instituto Nacional de Ciencias Penales (INACIPE) en la ciudad de México, donde la criminología verde se presenta como un enfoque contemporáneo a resolver. (Instituto Nacional de Ciencias Penales, 2019) y con ello, se realizó el Simposio de Criminología Verde, organizado por el INACIPE en la ciudad de México, de donde la corriente de la criminología que se identifica como criminología verde, se interesa por las afectaciones que se presentan del medio ambiente. (Instituto Nacional de Ciencias Penales, 2019).

Estos dos antecedentes educativos de generación y divulgación del conocimiento relativo a la criminología verde en México reducen la brecha entre la disciplina y el impacto de las actividades nocivas humanas en el medio ambiente. Sin embargo, no se encontraron más registros de cursos, diplomados, etc., en el tema, por lo que fue necesaria una revisión de los contenidos temáticos de los programas de formación profesional en la disciplina. Contenido de materias educativas relativas a crímenes ambientales en la enseñanza de la Criminología en el país a nivel educativo superior.

De acuerdo con la Asociación Nacional de Universidades e Instituciones de Educación Superior (ANUIES, 2018), en México existen un total de 184 programas educativos sobre Criminología, distribuidos en 96 universidades. De los 96 Instituciones de Educación Superior (IES) públicas y privadas en México que imparten la Licenciatura en Criminología, 8 son universidades públicas y 89 privadas, de las cuales, únicamente el $8.3 \%$ tienen dentro de su plan de estudios alguna materia relativa a las siguientes temáticas: Naturaleza, Medio Ambiente, Bioética, Desarrollo Sustentable/Sostenible y Ciencias Ambientales (Tabla 1).

En el estudio se identificó que las principales posturas de las materias tienden a abordar temáticas relativas a la naturaleza, los procesos biológicos y geológicos, etc., siendo la materia de desarrollo sustentable la que abona temáticas como los recursos naturales, economía, pobreza y otros aspectos demográficos, mientras que la bioética se orienta a la buena praxis forense; lo cual, coincide con lo establecido por González (2014) al hacer mención que la educación ambiental en México, ha sido promovida desde el sector de la naturaleza, por lo que puede observarse con bastante claridad que el enfoque para la enseñanza de las ciencias naturales domina la concepción de educación ambiental en la Secretaría de Educación Pública, sobre todo en el nivel básico, promoviendo que en los libros de texto de ciencias naturales y de geografía exista una equivalencia errónea entre ambiente y naturaleza que poco ha contribuido a ver la dimensión social de los problemas ambientales que obstruye la comprensión colectiva de los conflictos entre el hombre y la naturaleza.

Se identificó que las materias relacionadas al medio ambiente en la enseñanza criminológica han ocupado un lugar en la curricula de materias de "tronco común" por lo que el contenido de 


\begin{tabular}{|c|c|l|c|c|c|c|}
\hline \multicolumn{2}{|c|}{ Universidades } & \multicolumn{5}{|c|}{ Variables } \\
\hline Pública & Privada & $\begin{array}{l}\text { Naturaleza } \\
\text { y Medio } \\
\text { Ambiente }\end{array}$ & Ecología & $\begin{array}{c}\text { Desarrollo } \\
\text { Sustentable/ } \\
\text { Sostenible }\end{array}$ & Bioética & $\begin{array}{c}\text { Ciencias } \\
\text { Ambientales }\end{array}$ \\
\hline 8 & 88 & 2 & 0 & 3 & 1 & 2 \\
\hline
\end{tabular}

Fuente: Elaboración propia.

Nota: De la muestra de 96 universidades, 20 no facilitaron el plan de estudios por cuestiones de políticas internas.

Tabla 1. Materias relacionadas con temas ambientales en los planes de estudio de Licenciatura en Criminología en México.

Table 1. Subjects related to environmental issues in the study plans of the Degree in Criminology in Mexico.

esas materias es muy general y no permite una profundización crítica en la teoría y en la práctica que permita a los alumnos aplicar los conocimientos formativos de la disciplina en los distintos problemas ambientales del país y en consecuencia reduce la participación activa de recursos humanos como capital social en la atención y prevención de conductas antisociales con consecuencias en el medio ambiente.

Es importante resaltar que las materias relacionadas al medio ambiente encontradas en la formación de criminólogos se encuentran porcentualmente en universidades privadas con un $75 \%$ respecto a las universidades y centros de investigación de carácter público con un 25 $\%$, teniendo en cuenta que el mayor número de universidades que imparten la carrera son privadas y únicamente 5 son públicas (Figura 1).

Se identificaron materias en universidades de 6 estados (Chiapas, Puebla, San Luis Potosí, Tamaulipas, Nuevo León y Chihuahua), en los cuales tanto universidades públicas como privadas imparten criminología; en el caso de las universidades públicas, la materia de desarrollo sustentable es parte de la curricula de tronco común, pero han sido estas instituciones las que han tomado iniciativa de incorporar dentro del universo educativo superior los desafíos ambientales y la comprensión del desarrollo sustentable (Cantú, 2017), sin embargo, no pasa lo mismo con las IES privadas, ya que a menudo se encargan de "capacitar para el trabajo" dejando de lado la profundización teórica, aún así en los datos obtenidos son las que tienen una variedad de materias relacionadas al medio ambiente en criminología (Figura 2). Se encontró que no en todos los estados se aborda el tema planteado, lo cual es necesario, ya que México, tiene el cuarto lugar en biodiversidad a nivel mundial de acuerdo a los datos de la Comisión Nacional para el Conocimiento y Uso de la Biodiversidad (CONABIO, 2009) y los problemas de deforestación, crecimiento urbano no planificado, tráfico de especies, contaminación, etc., pueden ser analizados desde una postura criminológica que coadyuve en su prevención y regulación para cada estado de la república.

Además de acuerdo con la Secretaria de Medio Ambiente y Recursos Naturales (SEMARNAT) (2019) la educación ambiental debe mostrar la confluencia de todas las áreas disciplinarias y del conocimiento que contribuyen al análisis y comprensión de las relaciones que, a través de su historia, los hombres han establecido con su entorno.

Otro aspecto de relevancia identificado en el estudio es que persiste la confusión entre la criminología ambiental y la perspectiva verde de la criminología, ya que tres universidades tienen la materia de "criminología ambiental", de los 


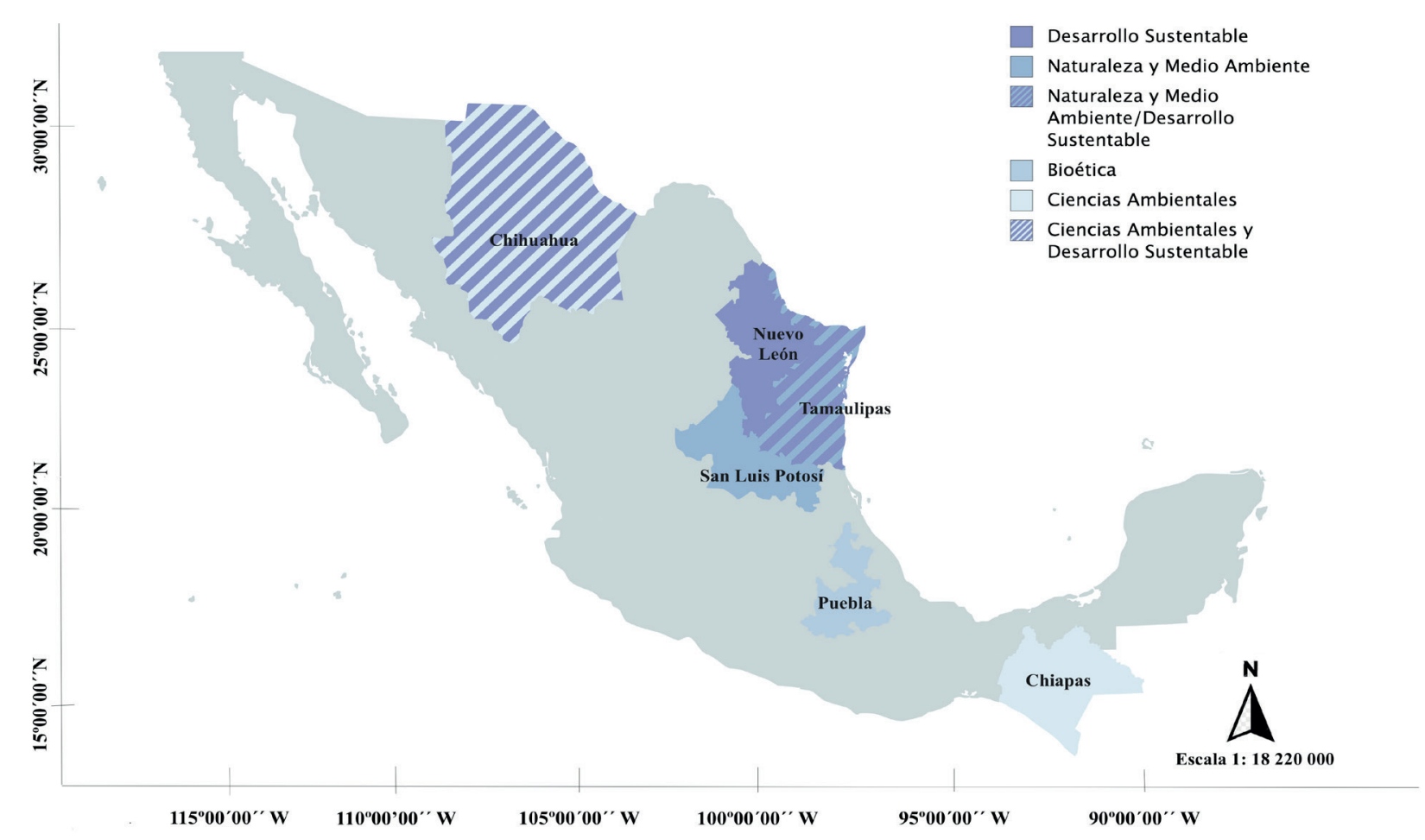

Fuente: Elaboración propia.

Figura 1. Materias relacionadas con temas ambientales en los planes de estudio de Licenciatura en Criminología en México.

Figure 1. Subjects related to environmental issues in the study plans of the Degree in Criminology in Mexico.

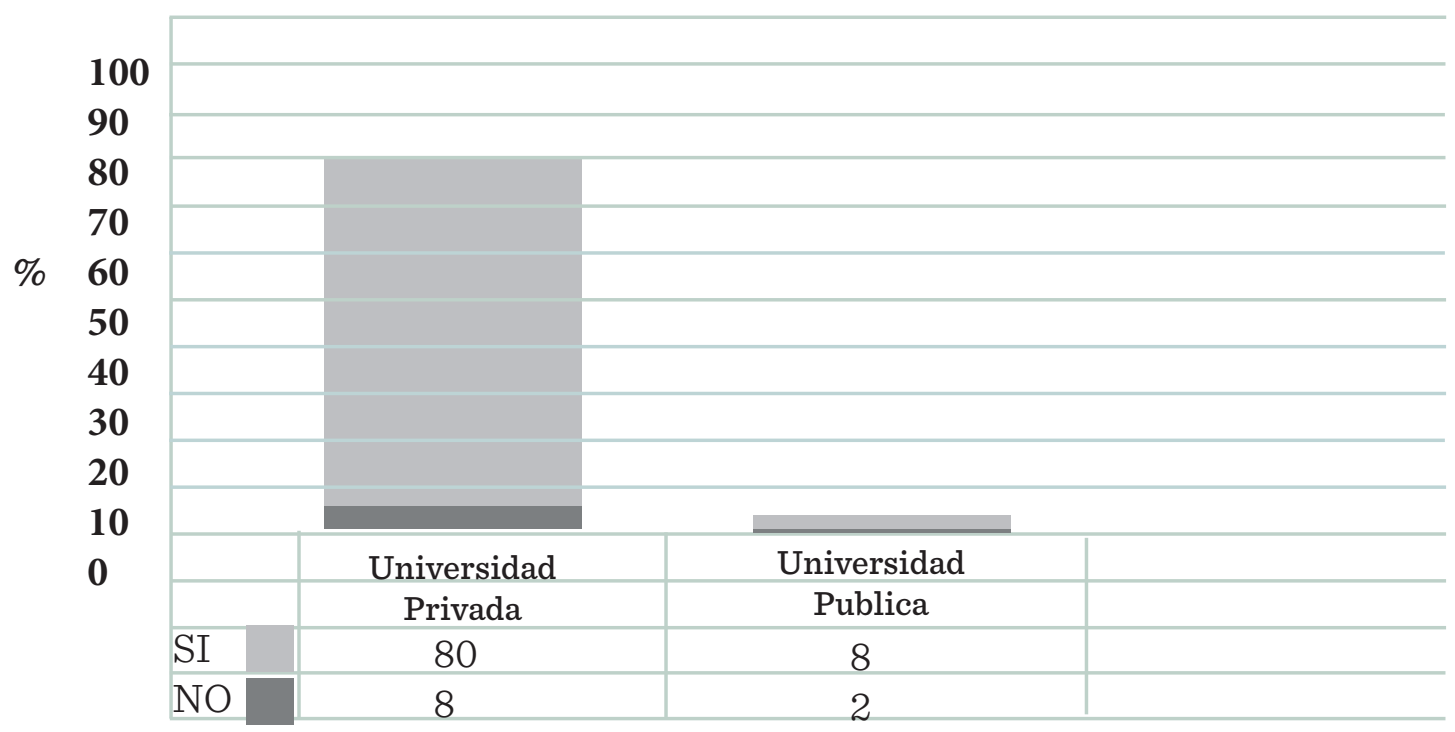

Fuente: Elaboración propia.

Figura 1. Presencia de materias relacionadas al medio ambiente en las Licenciatura en Criminología en las universidades en México.

Figure 1. Presence of subjects related to environment in the Degree in Criminology in the universities in Mexico. 
cuales, en dos la materias de "criminología ambiental" tiene contenido relativo a medio ambiente y a ecología del delito, lo que aumenta la confusión entre la criminología verde con relación a los crímenes ambientales y la ecología del delito que es parte de la investigación criminal. Esta confusión se ha prolongado a través de los últimos años, incluso se ha proyectado a trabajos de investigación científica, por lo que es imperativo discernir entre Criminología Ambiental y Criminología Verde (Tabla 2) a fin de que los trabajos de investigación y docencia vayan encaminados a fortalecer y diferenciar ambos aspectos importantes en la enseñanza criminológica.

Si bien, puede ser un fenómeno que se generó en la ausencia de una perspectiva verde de la criminología en México, la criminología ambiental fungió como un repositorio de los pro- blemas ambientales contemplados por la criminología y a través de los años se fue afianzando en la enseñanza; por lo que es imperativo que cada una de estos enfoques de la criminología se apropie de su objeto de estudio y se genere conocimiento adecuado y se difunda desde la investigación y la docencia.

Propuesta de una materia a nivel universitario que aborde la problemática de crímenes ambientales tomando como marco de referencia la Criminología Verde

Para atender a las necesidades planteadas anteriormente, se sugiere el siguiente contenido temático sobre Criminología Verde, para ser considerado en los planes de estudio universitarios en Criminología con el fin de contribuir a reforzar y fomentar la sensibilización, el conocimiento y la corresponsabilidad social so-
Criminología Verde (Green Criminology)

Es una perspectiva dentro de la Criminología que atiende a las actividades nocivas humanas y su impacto en el medio ambiente como una conducta antisocial.

Se ha definido como el trabajo criminológico que se enfoca sobre el daño ambiental (White, 2008). Analiza los daños ambientales desde una perspectiva criminológica, o la aplicación del pensamiento criminológico a los problemas ambientales (Hall et al., 2016).

Crimen Ambiental: (ecológico) es toda conducta antisocial que menoscaba la integridad ecológica y el patrimonio colectivo, de manera no controlada, y en consecuencia fuera de la ley, limitando las capacidades de productividad de las comunidades y las regiones (Carpio et al., 2018)

Ofrece un análisis teórico crítico de las acciones u omisiones humanas y sus consecuencias en el medio ambiente.

\section{Criminología Ambiental}

Es una rama de la criminología que estudia el espacio o lugar y el momento en que se ha cometido un delito (Pozuelo, 2018)

Plantea que los eventos delictivos deben entenderse como una confluencia de infractores, víctimas y objetivos del delito y normativas legales en escenarios específicos, ocurriendo en un momento y lugar concretos (Brantingham y Brantingham, 1991)

Ecología del crimen: las premisas pueden resumirse en tres puntos: la influencia del ambiente en la conducta delictiva, la no aleatoriedad de la distribución espacio-temporal del delito y la utilidad de los elementos anteriores en el control y prevención del delito (Wortley y Mazerolle, 2008)

Ofrece un conjunto de posibilidades en materia de lo que se denomina la prevención situacional del delito (San Juan, 2013).

Fuente: Elaboración propia con datos de los autores

Figura 1. Presencia de materias relacionadas al medio ambiente en las Licenciatura en Criminología en las universidades en México.

Figure 1. Presence of subjects related to environment in the Degree in Criminology in the universities in Mexico. 
bre crímenes ambientales en México desde la formación profesional de criminólogos.

\section{Criminología Verde}

1. Los niveles de interpretación de la Criminología

1.1. Crimen

1.2. Criminal

1.3. Criminalidad

1.4. Conceptos operaciones de orden explicativo

1.4.1. Causa

1.4.2. Factor

1.4.3. Móvil

1.4.4. Factor causal

2. Conceptos epistemológicos de la Criminología Verde

2.1. El concepto de crimen ambiental

2.2. Diferencias entre la criminología verde y la criminología ambiental

2.2.1. Crimen Ambiental

2.2.2. Ecología del crimen

2.3. Historia de la perspectiva verde

2.4. Factores criminógenos

2.5. Criminogenésis y criminodinámicas ambientales

2.5.1. Principales aportes de la perspectiva verde en la criminología

3. Los desafíos del Desarrollo Sustentable

3.1. Historia del Desarrollo Sustentable

3.2. Conceptos generales del Desarrollo Sustentable

3.3. Enfoques de Desarrollo Sustentable

3.3.1. Enfoque Económico

3.3.2. Enfoque Social

3.3.3. Enfoque Ambiental

3.3.4. Los Objetivos del Desarrollo Sostenible

4. El capital social

4.1. Concepto de capital social

$4.2 \mathrm{El}$ capital social en la formulación de políticas

5. El Capital natural

5.1. Concepto de capital natural

5.2. Riqueza natural de México

5.2.1. Biosfera

5.2.2. Flora y fauna

5.2.3. Ecosistemas

6. Legislación ambiental en México

6.1. Leyes y Códigos
6.1.1. Constitución Política de los Estados Unidos Mexicanos

6.1.2. Código Penal Federal

6.1.3. Ley General de Equilibrio Ecológico y Protección al Ambiente

6.1.4. Ley General de Vida Silvestre

6.2. Normas Oficiales Mexicanas en materia ambiental

6.2.1. Norma Oficial Mexicana 059- SEMARNAT

6.3. Tratados Internacionales

6.3.1. Convención sobre el Comercio Internacional de Especies Amenazadas de Fauna y Flora Silvestres (CITES)

6.3.2. Unión Internacional para la Conservación de la Naturaleza (IUCN)

6.3.3. Convenios Internacionales sobre medio ambiente

7. Los problemas ambientales

7.1. La situación actual del medio ambiente en México.

7.2. Revisión de políticas públicas en materia ambiental

7.3. Desafíos ambientales

7.3.1. El tráfico de especies

7.3.2. La contaminación atmosférica

7.3.3. La caza furtiva

7.3.4. Contaminación de mantos acuíferos

7.3.5. Desechos y basura

7.3.6. Pesca ilegal

7.3.7. Posesión ilegal de flora y fauna

7.3.8. Bioterrorismo

7.3.9. Contaminación Radiactiva

7.3.10. Sobreexplotación minera

7.3.11. Grupos vulnerables y problemas ambientales

7.3.12. Residuos químicos

7.3.13. Tala ilegal de bosques y selvas

8. La delincuencia organizada y sus consecuencias ambientales

8.1. El tráfico ilegal de especies

8.2. La deforestación y la siembra de droga

8.3. Desplazamiento geográfico de grupos vulnerables

8.4. Los problemas socioeconómicos en las localidades como consecuencia de eventos violentos.

9. Políticas Verdes

10. Tribunales Verdes

11. Elaboración de protocolos y estrategias 
11.1. Protocolos de actuación

11.2. Protocolos de prevención

11.3. Estrategias operativas en el monitoreo.

11.4. Estrategias operativas de actuación.

11.5. Estrategias de inteligencia en el monitoreo

11.6. Estrategias de inteligencia en la actuación.

12. Redacción de informes

12.1. Redacción de informes técnicos

\section{CONCLUSIONES}

La atención y prevención de los crímenes ambientales ha sido una tarea pendiente dentro del área de estudio de la Criminología en México. Sin embargo, la Criminología cuenta con todas las herramientas para el desarrollo de esta disciplina como ha venido ocurriendo en universidades de otros países desde la década de 1990, dado que la Criminología estudia las conductas antisociales para promover estrategias de prevención desde el enfoque social, considera que los crímenes ambientales son la consecuencia de actividades humanas e identifica los factores que determinan estas conductas (factores criminoimpelentes y factores criminorepelentes) y propone estrategias de prevención a través de la investigación científica, protocolos y estrategias para generar políticas públicas. Poco a poco, la enseñanza de la Criminología Verde se ha ido consolidando, a través de los años, dentro del marco de referencia de la Criminología formal y se ha ido adaptando a los nuevos fenómenos de criminalidad incluyendo aquellas asociadas con el medio ambiente., sin embargo, se evidencia la ausencia de contenido de problemas ambientales en la formación criminológica en las universidades en el país.

La criminología puede aportar, a través de sus metodologías, métodos e instrumentos para abordar propiamente los problemas de carácter ambiental y auxiliar en la identificación del origen de problemas ambientales (criminogenésis), la explicación de las dinámicas antisociales en materia ambiental (criminodinámica), en la construcción de estrategias de prevención, generación de protocolos de actuación, el análisis científico de los crímenes ambientales, el fortalecimiento de marcos regulatorios, así como en el apoyo a las estrategias gubernamentales de educación ambiental y capacitación a las fuerzas del Estado y de corresponsabilidad social.

\section{REFERENCIAS}

Agnew, R. (2012). Dire Forecast: A Theoretical Model of the Impact of Climate Change on Crime. Journal of Theoretical Criminology, 16(1), 21-42. Recuperado de https://journals.sagepub.com/ doi/abs/10.117r/1362480611416843?journalCode=tcra. Fecha de Consulta: 29 de marzo de 2019.

Alvarado, I. (2012). Delincuencia organizada ambiental en México, una nueva manifestación criminal del tráfico de especies. Revista Criminalidad, (54)1, 283-311. Recuperado de http://www. scielo.org.co/pdf/crim/v54n1/v54n1a05.pdf. Fecha de Consulta: 18 de abril de 2019.

Aragonés, J., Izurieta, C. y Raposo, G. (2003). Revisando el concepto de desarrollo sostenible en el discurso social. Revista Psicothema. 15(2), 221-226. Recuperado de http://www.psicothema. com/pdf/1049.pdf. Fecha de Consulta: 16 de marzo de 2019.

Arroyo, I. y Wyatt, T. (2018). Criminología verde en México. Recuperado de https://www.crim. unam.mx/web/sites/default/files/Muestra_Criminolog\%C3\%ADa\%20verde_0.pdf. Fecha de Consulta: 18 de abril de 2019.

Asociación Nacional de Universidades e Instituciones de Educación Superior (2018). Anuarios Estadisticos de Educación Superior. Recuperado de http://www.anuies.mx/informacion-y-servicios/informacion-estadistica-de-educacion-superior/anuario-estadistico-de-educacion-superior. Fecha de Consulta: 29 de marzo de 2019.

Beirne, P. (1999). For a Nonspeciesist Criminology: Animal Abuse as an Object of Study. Journal of Criminology, 37(1), 117-148. Recuperado de https://onlinelibrary.wiley.com/doi/pdf/10.1111/j.1745-9125.1999.tb00481.x. Fecha de Consulta: 16 de marzo de 2019.

Beirne, P. and South, N. (2007). Issues in Green Criminology: Confronting Harms Against Environments, Humanity and Other Animals. Ed. Cullompton: Willan Publishing. 340 p.

Benton, T. (2007). Ecology, community and justice: The meaning of green. Issues in Green Criminology. Cullompton: Willan Publishing, pp. 3-31. 
Bisschop, L. (2012). Is It All Going to Waste? Illegal Transports of E-waste in a European Trade Hub. Crime. Law and Social Change, 58(3), 221-249. Recuperado de https://link.springer. com/article/10.1007/s10611-012-9383-0. Fecha de Consulta: 16 de marzo de 2019.

Brantingham, P. y Brantingham, P. (1991). Environmental Criminology. Publicaciones Sage, 264p.

Cantú, P. (2017). Instituciones de Educación Superior y la sustentabilidad. Revista Ciencia UANL. Año 20 (6), pp. 20-24. Recuperado de: https://drive.google.com/file/d/1uCQ6ai5knqHZTzrcuIEHQD4QHa9hTqSX/view

Carpio, J., Vargas, C., Villarreal, K. y Meraz, M. (2018). Las redes sociales como factor criminógeno de la venta ilegal de especies en Tamaulipas (México): el caso de Facebook. Revista CienciaUAT.13(1), 19-34. Recuperado de http://www. revistaciencia.uat.edu.mx/index.php/CienciaUAT/article/view/972/466. Fecha de Consulta: 18 de abril de 2019.

Carrabine, E., Iganski, P., Lee, M. Plummer, P. and South, N. (2004). Criminology, A Sociological Introduction. Recuperado de https://www.hrstud. unizg.hr/_download/repository/Eamonn_Carrabine\%2C_Maggy_Lee\%2C_Nigel_South\%2C_ Pam_Cox\%2C_Ken_Plummer_Criminology_A_ Sociological_Introduction_2009.pdf. Fecha de Consulta: 31 de marzo de 2019.

Carson, R. (1962). Silent Spring. Revista mexicana de ciencias forestales, 3(14). Recuperado de http://www.scielo.org.mx/pdf/remcf/v3n14/ v3n14a1.pdf. Fecha de Consulta: 3 de marzo de 2019.

Clarke, R. and Timberlake, L. (1982). Estocolmo más diez promesas, ¿̇promesas? La década desde la Conferencia de las Naciones Unidas para el Medio Ambiente de 1972. Conferencia de las Naciones Unidas sobre el Medio Ambiente. Ed. Earthscan. Londres. 75 p.

Clifford, M. (1998). Environmental crime: enforcement, policy, and social responsibility. Gaithersburg, Md.: Aspen Publishers. 532 p.

Croall, H. (2009). White Collar Crime, Consumers and Victimization. Crime, Law and Social Change, 51(1), 127-146. Recuperado de https:// hal.archives-ouvertes.fr/hal-00478394/docu- ment. Fecha de Consulta: 16 de marzo de 2019.

Eman, K., Mesko, G. and Fields, C. (2009). Crimes against the Environment: Green Criminology and Research Challenges in Slovenia. Varstvoslovje Journal of Criminal Justice and Security, 11(4), 574-592. Recuperado de https://s3.amazonaws.com/academia.edu.documents/43125131/ vs-2009-4-eman-mesko-fields.pdf?AWSAccessKeyId=AKIAIWOWYYGZ2Y53UL3A\&Expires $=1554021672 \&$ Signature $=i N L \% 2 B d I X X U U t-$ bhRV2V16ZmCXLbrA\%3D\&response-content-disposition=inline\%3B\%20filename\%3DCrimes_against_the_environment_green_cri. pdf. Fecha de Consulta: 30 de marzo de 2019.

Eschenhagen, M. (2011). Contexto y exigencias a las ciencias sociales para afrontar los problemas ambientales. Polis Revista Latinoamericana, 30(21). Recuperado de https://journals.openedition.org/polis/2324. Fecha de Consulta: 30 de marzo de 2019.

Fernández, L. y Gutiérrez, M. (2013). Bienestar Social, Económico y Ambiental para las Presentes y Futuras Generaciones. Revista Información Tecnológica. Vol. 24 (2), pp. 121-130. Recuperado de: https://scielo.conicyt.cl/pdf/infotec/v24n2/ art13.pdf.

Gibbs, C., Gore, M., McGarrell, E. and Rivers, L. (2010). Introducing Conservation Criminology: Toward Interdisciplinary Scholarship on Environmental Crimes and Risks. The British Journal of Criminology, 50(1), 124-144. Recuperado de http://citeseerx.ist.psu.edu/viewdoc/download?doi=10.1.1.819.1629\&rep=rep1\&type=pdf. Fecha de Consulta: 16 de marzo de 2019.

Giordan, A y Souchon, C. (1995). La Educación Ambiental, Guía Práctica. Ed. Díada, Sevilla. 227 p.

Global Footprint Network. (2013). Huella mundial: ¿Encajamos en el planeta? Global Footprint Network. Recuperado de https://www.footprintnetwork.org/our-work/ecological-footprint/\#worldfootprint. Fecha de Consulta: 3 de marzo de 2019.

González, E. (2014). Atisbando el desarrollo conceptual de la educación ambiental en México. Horizonte Sanitario, 2(1), 34-43. Recuperado de https:/www.researchgate.net/ publication/320512476_Atisbando_el_desarro- 
llo_conceptual_de_la_educacion_ambiental_en_ Mexico. Fecha de Consulta: 22 de abril de 2019.

Groombridge, N. (1998). Masculinities and Crimes Against the Environment. Journal of Theoretical Criminology. 2 (2), 249-267. Recuperado de https://journals.sagepub.com/doi/10.1177/13624 80698002002006.

Hall, M. (2013). Victims of Environmental Harm: Rights, Recognition and Redress Under National and International Law. Abingdon: Routledge. $202 \mathrm{p}$.

Hall, M., Maher, J., Nurse, A., Potter, G., South, N. and Wyatt, T. (2016) Introduction: Green criminology in the 21st century. In: Greening Criminology in the 21st Century. Taylor \& Francis, pp. 1-8. Recuperado de: http://nrl.northumbria. ac.uk/36570/.

Hardin, G. (1968). La Tragedia de los Comunes. Revista Science, 162 (1968), 1243-1248. Recuperado de http://www.arschile.cl/moodledata/2/ Mod4/TragediaComunes.pdf. Fecha de Consulta: 3 de marzo de 2019.

Hauck, M. (2008). Rethinking Small-scale Fisheries Compliance. Journal of Marine Policy, 32(4), 635-642. Recuperado de https://ideas. repec.org/a/eee/marpol/v32y2008i4p635-642. html. Fecha de Consulta: 16 de marzo de 2019.

Jarrell, M. and Ozymy, J. (2012). Real Crime, Real Victims: Environmental Crime Victims and the Crime Victims' Rights Act (CVRA). Journal of Crime, Law and Social Change, 58(4), 373-389. Recuperado de https://link.springer.com/article/10.1007/s10611-012-9394-x. Fecha de Consulta: 30 de marzo de 2019.

Katz, R. (2010). The Corporate Crimes of Dow Chemical and the Failure to Regulate Environmental Pollution. Critical Criminology, 18(4), 295-306. Recuperado de https://student. cc.uoc.gr/uploadFiles/181-\%CE\%95\%CE\%93\%CE\%9A\%CE\%9A339/Katz\%20Corporate\%20 crimes\%20of\%20dow\%20chemical.pdf. Fecha de Consulta: 30 de marzo de 2019.

Ley General de Equilibrio Ecológico y Protección al Ambiente (2018). Diario Oficial de la Federación del 5 de junio de 2018. Cámara de Diputados del Honorable Congreso de la Unión. Recuperado de http://www.diputados.gob.mx/ LeyesBiblio/pdf/148_050618.pdf. Fecha de Con- sulta: 15 de marzo de 2019.

Ley Ambiental de Protección a la Tierra en el Distrito Federal (2017). Última reforma publicada en la Gaceta Oficial de la Ciudad de México, el 08 de septiembre de 2017. Recuperado de http:// www.paot.org.mx/centro/leyes/df/pdf/2017/ LEY_AMBIENTAL_PROTECCION_TIERRA_08_09_2017.pdf. Fecha de Consulta: 22 de abril de 2019.

Lynch, M. (1990). The Greening of Criminology: A Perspective for the 1990's. The Critical Criminologist. Ed. Routledge. 614 p. Recuperado de https://www.taylorfrancis.com/books/e/9781351564977/chapters/10.4324/9781315093390-7. Fecha de Consulta: 30 de marzo de 2019.

Lynch, M. and Stretesky, P. (2001). Toxic crimes: Examining corporate victimization of the general public employing medical and epidemiological evidence. Critical Criminology. 10(3), 153172. Recuperado de https://www.researchgate. net/profile/Michael_Lynch24/publication/226205597_Toxic_Crimes_Examining_ Corporate_Victimization_of_the_General_Public_Employing_Medical_and_Epidemiological_ Evidence/links/559f050108ae03c44a5ce19b. pdf. Fecha de Consulta: 30 de marzo de 2019.

Martínez, R. (2010). La importancia de la educación ambiental ante la problemática actual. Revista Electrónica Educare, 14(1), 97111. Recuperado de https://www.redalyc.org/ html/1941/194114419010/. Fecha de Consulta: 30 de marzo de 2019.

Márquez, G. (2002). Ecología y Cultura: Cambio ambiental, evolución biológica y evolución cultural. Politeia, 28, 41-56. Recuperado de http:// www.idea.unal.edu.co/publica/docs/ecolg_cultura.pdf. Fecha de Consulta: 18 de abril de 2019.

Montemayor, M. (2003). Educación Ambiental. Medio Ambiente y Desarrollo Sustentable. Universidad Autónoma de Tamaulipas. Segunda Edición, Colección Misión XXI, pp. 205-225.

Nurse, A. (2013). Privatizing the Green Police: The Role of NGO's in Wildlife Law Enforcement. Journal of Crime, Law and Social Change, 59 (3), 305-318. Recuperado https://link.springer.com/ article/10.1007/s10611-013-9417-2. Fecha de Consulta: 16 de marzo de 2019. 
Organización de las Naciones Unidas. (1972). Declaración de Estocolmo sobre el medio ambiente humano. Conferencia de las Naciones Unidas sobre el Medio Ambiente Humano. 4 p. Recuperado de: http://www.ordenjuridico.gob.mx/TratInt/ Derechos\%20Humanos/INST\%2005.pdf

Organización de las Naciones Unidas. (2000). Agenda 21. División de las Naciones Unidas para el Desarrollo Sostenible. 690 p. Recuperado de: http://biblioteca.semarnat.gob.mx/janium/ recursos/224844/Contenido/H\%20programas/23\%20Agenda\%2021.pdf

Otero, I. (2000). Paisaje y Educación Ambiental. Revista Observatorio Medioambiental, 3, 3550. Recuperado de https://revistas.ucm.es/index. php/OBMD/article/view/OBMD0000110035A. Fecha de Consulta: 16 de marzo de 2019.

Pecar, J. (1981). Crimen ecológico y criminología. Revista de Criminalística y Criminología, 34 (1),33-45.Recuperado de https://www.policija.si/ images/stories/Publikacije/RKK/PDF/1981/01/ RKK1981-01_JanezPecar_EkoloskaKriminaliteta.pdf. Fecha de Consulta: 31 de marzo de 2019.

Pecar, J. (1988). La opinión pública "criminológica”. Actas de las discusiones científicas, 48(1), 105-125.

Peralta, L. (2018). Los tribunales verdes en México. La sustentabilidad en la Ley Ambiental y la construcción de un nuevo paradigma institucional. Ed. Colofón, México. 172 p.

Perdomo y Escobar. (2011). La investigación en rse: una revisión del magnament. Cuadernos de Administración, 24(43), 193-219. Recuperado de http://www.scielo.org.co/pdf/cadm/v24n43/ v24n43a10.pdf. Fecha de Consulta: 21 de abril 2019.

Pozuelo, C. (2018). Criminología Ambiental. La prevención del delito a través del estudio del diseño urbano. Grado en Criminología curso 2017-2018. Universitat Oberta de Catalunya. 38 p. Recuperado de: http://openaccess.uoc.edu/webapps/o2/bitstream/10609/75765/6/cpozuelofTFG0118memoria.pdf.

Programa de las Naciones Unidas para el Ambiente (1975). La Carta de Belgrado: un marco general para la educación ambiental. Seminario Internacional de Educación Ambiental de UNESCO-PNUMA. 5 p. Recuperado de: https://unes- doc.unesco.org/ark:/48223/pf00000177772_spa

Ramírez, J. y Ramírez, G. (2004). Educación ambiental: conocer, valorar y conservar el medio. Revista Ecofronteras, 20, 38-40. Recuperado de. Disponible en: http://revistas.ecosur.mx/ecofronteras/index.php/eco/search/search. Fecha de Consulta: 16 de marzo de 2019

Rodríguez, L. (2015). Criminología. Ed. Porrúa. $562 \mathrm{p}$.

Ruggiero, V. and South, N. (2010). Green Criminology and Dirty Collar Crime. Critical Criminology, 18(4), 251-262. Recuperado de https:// student.cc.uoc.gr/uploadFiles/181-\%CE\%95\%CE\%93\%CE\%9A\%CE\%9A339/Ruggiero\%20 and\%20Kramer\%20Green\%20criminology\%20 and\%20dirty\%20collar\%20crime.pdf. Fecha de Consulta: 11 de marzo de 2019

San Juan, C. (2013). Criminología ambiental: un área en expansión. Tribuna de Actualidad. Vol. 1, pp. 33-38. Recuperado de: https://www.researchgate.net/publication/318234773_Criminologia_Ambiental_un_area_en_expansion

Sauvé, L. (2005). Una cartografía de las corrientes en educación ambiental. Educación ambiental, Investigación y desafíos. Porto Alegre: Artmed, pp. 17-46. Recuperado de http://www. ecominga.uqam.ca/PDF/BIBLIOGRAPHIE/ GUIDE_LECTURE_3/1/2.Sauve.pdf. Fecha de Consulta: 11 de marzo de 2019

Sollund, R. (2008). Global Harms: Ecological Crime and Speciesism. Nova Science Publishers Inc. United Kingdom. 228 p.

Sosa, J. (2011). Aplicación de la Ley para el Combate del Tráfico Ilegal de Vida Silvestre en México: El caso de Charco. Revista Therya, 2 (3), 245-262. Recuperado de http://www.scielo.org. mx/pdf/therya/v2n3/v2n3a5.pdf. Fecha de Consulta: 18 de abril de 2018.

South, N. and Brisman, A. (2013). Routledge International Handbook of Green Criminology. Routledge. New York. 450 p.

Takemura, N. (2015). Dynamic Complexity of Environmental Crime: Some Aspects of Applied Green Criminology. Thirteenth United Nations Congress on Crime Prevention and Criminal Justice. 81 p. Recuperado de https://www.unodc.org/ documents/congress/background-information/ Individual_Experts/2015_UN_Congress_report_ 
IE_N.Takemura.pdf. Fecha de Consulta: 3 de marzo de 2019.

Thompson, S. y Barton, M. (1994). Actitudes ecocéntricas y antropocéntricas hacia el medio ambiente. Revista de psicología ambienta. 14 (2), 149-157. Recuperado de https://www. sciencedirect.com/science/article/abs/pii/ S0272494405801689. Fecha de Consulta: 3 de marzo de 2019.

Toledo, V. (2015). El ecocidio en México. La batalla final es por la vida. Ed. Grijalbo. 176 p.

Van Solinge, T. (2010). Deforestation Crimes and Conflicts in the Amazon. Critical Criminology 18(4), 263-27\%. Recuperado de https://www. peacepalacelibrary.nl/ebooks/files/352205865. pdf. Fecha de Consulta: 3 de marzo de 2019.

Walters, R. (2006). Crime, Bio-Agriculture and the Exploitation of Hunger. The British Journal of Criminology, 46(1), 26-45. Recuperado de https:// www.jstor.org/stable/23639328?mag=gmos-inequality-world-hunger\&seq=1\#page_scan_tab_ contents. Fecha de Consulta: 3 de marzo de 2019.

White, R. (2008). Crimes Against Nature: Environmental Criminology and Ecological Justice. Ed. Cullompton: Willan. 328 p.

Wortley, R. and Mazerolle, L. (2008). Environmental Criminology and Crime Analysis: situating the theory, analytic approach and application. En R. Wortley \& L. Mazerolle (eds.): Environmental Criminology and Crime Analysis. Ed. Willan Publising, pp. 1-18. Recuperado de: https://www. researchgate.net/publication/29468939_Environmental_Criminology_and_Crime_Analysis_ Situating_the_Theory_Analytic_Approach_and_ Application.

Wyatt, T. (2012). Green Criminology and Wildlife Trafficking: The Illegal Fur and Falcon Trades in Russia Far East. Saarbrucken: LAP Lambert Academic Publishing. 288 p. 